\title{
Structure control of a zinc tetraphenylporphyrin thin film by vapor annealing using fluorine containing solvent
}

\section{AUTHOR(S):}

Tomita, Kazutaka; Shioya, Nobutaka; Kise, Ryuma; Shimoaka, Takafumi; Yoshida, Hiroyuki; Koganezawa, Tomoyuki; Eda, Kazuo; Hasegawa, Takeshi

\section{CITATION:}

Tomita, Kazutaka ...[et al]. Structure control of a zinc tetraphenylporphyrin thin film by vapor annealing using fluorine containing solvent. Thin Solid Films 2018, 665: 85-90

\section{ISSUE DATE:}

2018-11-01

URL:

http://hdl.handle.net/2433/234712

\section{RIGHT:}

(c) 2018 This manuscript version is made available under the CC-BY-NC-ND 4.0 license

http://creativecommons.org/licenses/by-nc-nd/4.0/; The full-text file will be made open to the public on 1 November 2020 in accordance with publisher's 'Terms and Conditions for Self-Archiving'.; この論文は出版社版でありません。引 用の際には出版社版をご確認ご利用ください。; This is not the published version. Please cite only the published version. 


\section{Structure Control of a Zinc Tetraphenylporphyrin}

\section{Thin Film by Vapor Annealing Using Fluorine}

\section{Containing Solvent}

Kazutaka Tomita, ${ }^{a}$ Nobutaka Shioya, ${ }^{a}$ Ryuma Kise,${ }^{a}$ Takafumi Shimoaka, ${ }^{a}$ Hiroyuki

Yoshida, ${ }^{b, c}$ Tomoyuki Koganezawa, ${ }^{d}$ Kazuo Eda, ${ }^{e}$ and Takeshi Hasegawa*a

${ }^{a}$ Laboratory of Chemistry for Functionalized Surfaces, Division of Environmental

Chemistry, Institute for Chemical Research, Kyoto University, Gokasho, Uji, Kyoto 611-0011, Japan

${ }^{\mathrm{b}}$ Graduate School of Engineering and ${ }^{\mathrm{c}}$ Molecular Chirality Research Center, Chiba University, 1-33 Yayoi-cho, Inage-ku, Chiba 263-8522, Japan

d Japan Synchrotron Radiation Research Institute, 1-1-1 Kouto, Sayo-cho, Sayo-gun, Hyogo 679-5198, Japan

${ }^{\mathrm{e}}$ Department of Chemistry, Graduate School of Science, Kobe University, 1-1 Rokkodai, Nada-ku, Kobe, Hyogo 657-8501, Japan 
Abstract: The solvent vapor annealing (SVA) technique is one of the useful post processing techniques of a thin film, which is an alternative technique of the thermal annealing one. SVA has a great advantage that the molecular rearrangement in the film is made moderately by employing an appropriate solvent without the sample heating. The moderate processing is expected to yield a benefit that the molecular coalescence would be suppressed, which would readily keep the continuous surface topography of the film during the annealing, and another benefit that a metastable structure would be obtained. To make the best use of the SVA-specific characteristics, in the present study, a material having a metastable structure is chosen. The sample is zinc tetraphenylporphyrin (ZnTPP) that yields a metastable triclinic crystal structure, which can easily be converted to a monoclinic crystal structure by thermal annealing. A triclinic-structure film of ZnTPP by the combination of a wet process and the thermal annealing has thus never been reported. By choosing a fluorine-containing solvent, which has a low affinity to ZnTPP, a triclinicstructure film has first been obtained by a wet process while the surface continuity is protected.

Keywords: Solvent vapor annealing, Two-dimensional grazing incidence X-ray diffraction, p-polarized multiple-angle incidence resolution spectrometry, Molecular orientation, Porphyrin, Fluorine containing solvent, Organic semiconductor, Thin film 


\section{Introduction}

To improve the performance of organic thin-film devices, structural control of the organic semiconductor layer is a crucial technology, which has extensively been studied thus far [1-14]. In particular, an ordered thin film with a high crystallinity is needed for improving both exciton diffusion length and charge transfer [7,15]. As a useful technique for improving the crystallinity and the molecular orientation of an organic semiconductor layer, a thermal annealing and a solvent vapor annealing (SVA) are often employed [4,16-20].

A thermal annealing is the most commonly used as a post-processing technique because of the technical simplicity that the crystallinity and the molecular orientation of the thin film can be improved simply by heating the organic semiconductor layer. Heating, however, sometimes causes a side effect of structural deterioration of the semiconductor layer due to unexpected grain growth and coalescence of the grains, which induces discontinuous surface topography [21]. For example, a spin-coated film of zinc tetraphenylporphyrin (ZnTPP; Chart 1a) cannot keep the continuous surface topography after a thermal annealing at $100^{\circ} \mathrm{C}$ as found in Fig. 1a and b. Heating sometimes induces chemical degradation such as oxidation $[22,23]$ and decomposition [24] of the compound, which makes the device performance poorer [23,24]. 
To avoid these side effects via heating, SVA can be an alternative annealing technique. By employing SVA, the crystallinity and the molecular orientation of the thin film can be improved at ambient temperature by exposing the organic semiconductor layer to the solvent vapor $[4,16-20]$. Although SVA can easily be performed, choosing an appropriate annealing solvent is difficult. For example, when a good solvent of chloroform (Chl; Chart $1 \mathrm{~b})$ is employed for the annealing of the spin-coated thin film of $\mathrm{ZnTPP}$, the molecules in the annealed film are coalesced, which makes the film topography discontinuous as shown in Fig. 1c. The coalescence implies that the ZnTPP molecules are made to have an unnecessarily high molecular mobility after the solvation by Chl.

Another side effect of using a good solvent is that the annealed molecules attain the thermodynamically stabilized crystal structure represented by the monoclinic crystallite with the herringbone packing of ZnTPP [25,26] (Fig. 2a). In other words, a metastable crystal structure is passed by due to the large molecular mobility induced by the solvation. For example, in a ZnTPP thin film, the metastable triclinic phase with the face-to-face stacking [27] (Fig. 2b) is difficult to be prepared by a wet process using a good solvent [25]. Although the triclinic one can be obtained by the vapor deposition technique [28], a wet process is better when considering the merit of efficient mass- 
production.

Crystal structures having a herringbone packing or face-to-face stacking are found in other organic semiconductor materials such as phthalocyanine [29] and its derivative [30], which have a similar macrocycle molecular structure. The performance of the device is directly influenced by the molecular packing: the triclinic crystal with the faceto-face stacking of the phthalocyanine skeleton is calculated to have a higher carrier mobility than the monoclinic crystal with the herringbone packing [30]. To have the face-to-face stacking, the triclinic crystal structure of ZnTPP is a good candidate for the device development.

To obtain the metastable triclinic crystal phase by a wet process without breaking the continuous surface topography, an appropriate solvent for SVA should be searched for. For this purpose, a solvent with an appropriately weak molecular affinity to the film compound should be appropriate.

The molecular affinity can be interpreted as the molecular interactive forces such as hydrogen bonding, dispersion force and dipole-dipole interactions [31]. For the case of ZnTPP having phenyl groups, the intermolecular interaction is mostly driven by the dispersion force [32], which is common to Chl [33]. This is why Chl works as a good solvent for ZnTPP in fact. Since the dispersion force is related to a hydrocarbon part and 
a large-radius element like chlorine, in the present study, a solvent having some $\mathrm{C}-\mathrm{F}$ bonds should be used considering a smaller molecular polarizability of the $\mathrm{C}-\mathrm{F}$ bond, which yields a weak dispersion force [31,34]. In the present study, HCFC-225 (see Experimental; Chart 1c) is chosen for this reason. As expected, this solvent exhibited a poor solubility for ZnTPP.

As a result, the metastable triclinic phase of ZnTPP has readily been obtained as the major component of the SVA treated film, and the surface continuity is kept without a significant damage. This is the first report that the triclinic crystal of this compound is obtained in a continuous wet-processed film to our knowledge. The molecular structure in the film is analyzed in detail in terms of both crystal structure and the molecular orientation by using the grazing incidence X-ray diffraction technique equipped with a two-dimensional array detector (2D-GIXD) and infrared (IR) p-polarized multiple-angle incidence resolution spectrometry (pMAIRS [35-37]) technique, respectively.

\section{Experimental methods}

2.1 Materials: Zinc (II) tetraphenylporphyrin (ZnTPP) (Chart 1a) was purchased from Tokyo Chemical Industry Co. Ltd. (Tokyo, Japan), and it was used without further purification. Chloroform (Chl) (Chart $1 \mathrm{~b}$ ) was the ACS spectrophotometric grade with a 
purity of $\geq 99.8 \%$, which was purchased from Sigma-Aldrich (St. Louis, MO, USA). Hydrochlorofluorocarbon (HCFC)-225 (Chart 1c) was purchased from Wako Pure Chemical Industries, Ltd (Osaka, Japan).

2.2 Film Preparations: The silicon substrate, which was provided by Valqua FFT Inc.

(Tokyo, Japan) was sonicated for 60 seconds in each pure water, ethanol, acetone, and 1,2-dichloroethane, successively. The spin-coated films were prepared by dropping $20 \mu \mathrm{L}$ of a Chl solution of ZnTPP at $10.0 \mathrm{mM}$ onto the substrate spun at $2000 \mathrm{rpm}$. All films were prepared on a rectangle silicon substrate $\left(40 \times 20 \mathrm{~mm}^{2}\right)$ in ambient air $\left(25^{\circ} \mathrm{C}\right)$. For the SVA treatment, the coated layer on the substrate was faced down to the solvent surface with a distance of ca. $5 \mathrm{~mm}$ in an open system. The exposure time to the HCFC-225 vapor is $12 \mathrm{~h}$ at ambient temperature, which is much longer than the conventional annealing time $[4,16-19]$ for attaining the thermodynamic equilibrium.

2.3 Atomic Force Microscope Measurements: Atomic force microscopy (AFM) measurements were performed on a Seiko Instruments (Chiba, Japan) NanoNavi IIs probe station equipped with a probing microscope unit (Nanocute), which was set on an antivibration stage. The dynamic force mode was employed for the scanning of the surface topography. The force constant and resonance frequency of the silicon cantilever are 19 $\mathrm{N} \mathrm{m}^{-1}$ and $143 \mathrm{kHz}$, respectively. 
2.4 2D-GIXD measurements: 2D-GIXD experiments were carried out at the beamline, BL46XU, of a synchrotron radiation facility "SPring-8" (Harima, Hyogo, Japan). A radiated X-ray having the energy of $12.39 \mathrm{keV}(\lambda=0.1 \mathrm{~nm})$ was irradiated on the sample at a fixed angle of incidence of $0.12^{\circ}$ from the surface parallel, and the diffracted X-ray was led through a Huber (Rimsting, Germany) diffractometer to have diffraction patterns that were detected by a Pilatus 300K (Baden-Dättwil, Switzerland) two-dimensional imaging detector.

2.5 pMAIRS measurements: IR pMAIRS spectra were measured by using a Thermo Fischer Scientific (Madison, WI, USA) Nicolet 6700 FT-IR spectrometer equipped with a Thermo Fisher Scientific (Yokohama, Japan) automatic MAIRS equipment (TN101500). The light source was made modulated IR light with a frequency of $60 \mathrm{kHz}$. The ppolarized light was obtained by passing through a PIKE Technologies (Madison, WI, USA) manual polarizer (090-1500). The p-polarized IR light was transmitted through the sample substrate with the angle of incidence changing from $9^{\circ}$ to $44^{\circ}$ by $5^{\circ}$ steps [38]. The transmitted light was detected by a mercury-cadmium-telluride (MCT) detector, which is cooled for more than $2 \mathrm{~h}$ by liquid $\mathrm{N}_{2}$ in advance. The detected signal was accumulated 500 times for each angle. The IP (in-plane) and OP (out-of-plane) spectra were simultaneously obtained by pMAIRS analysis [39]. The IP spectrum exhibits the 
parallel component of a transition moment to the substrate; whereas the OP spectrum is for the perpendicular component to the substrate, which is the surface selection rule of the pMAIRS technique. When the optimum incident angles are employed, the orientation angle of a transition moment is readily calculated by the band intensity ratio of the IP to OP spectra $\left(A_{\mathrm{IP}} / A_{\mathrm{OP}}\right)$ using Equation (1) [40]

$$
\phi=\tan ^{-1} \sqrt{\frac{2 A_{\mathrm{IP}}}{A_{\mathrm{OP}}}}
$$

\section{Results and Discussion}

Fig. 3 shows AFM images of the surface topography at different stages in the film preparation process. Fig. 3a and $\mathrm{b}$ show the surface image of a bare silicon substrate and an as-spun film on the substrate, respectively. In Fig. 3b, a continuous flat film is readily obtained without forming apparent crystalline grains, which is a typical feature of an amorphous film [41,42]. In fact, in the 2D-GIXD measurement, no peaks are found in the diffraction pattern of the film (Fig. 4a). This clearly indicates that the film has an amorphous structure. The film has a very flat surface with a root mean square (rms) roughness of $0.28 \mathrm{~nm}$. This is even flatter than that of the bare silicon substrate $(\mathrm{rms}=$ $1.18 \mathrm{~nm})$.

Fig. 3c is the surface image after the SVA with the use of HCFC-225 (denoted as 
HCFC-SVA). The ZnTPP film after HCFC-SVA readily keeps the continuous surface topography in Fig. 3c, which has not been obtained by the conventional thermal annealing or SVA using Chl (denoted as Chl-SVA). Since HCFC-225 has a low molecular affinity, the ZnTPP molecules in this film should have a low mobility because of weak solvation. As a result of having a low mobility, the moderate aggregation is induced without breaking the continuous topography.

When the molecules have a low mobility on the substrate, the metastable structure is expected to be obtained in the film. To investigate the crystal structure generated in the ZnTPP thin film induced by HCFC-SVA, 2D-GIXD measurements are performed.

After the HCFC-SVA treatment, on the other hand, the diffraction pattern of the film is drastically changed to have many strong peaks (Fig. 4b), which clearly indicates that the molecules are highly crystallized. By referring to the calculated X-ray powder diffraction patterns of a single crystal [25-27], as expected, most of these peaks are readily assigned to the metastable triclinic crystal structure (denoted by ' $\mathrm{T}$ ' in Fig. 4; CCDC Refcode: ZZZTAY02 [27]), as well as the two monoclinic crystal structure (CCDC Refcode: ZZZTAY03 [26] and ZNTPOR03 [25]) denoted by 'Ma' and 'Mb' respectively. There are many $\mathrm{T}$ peaks with dominantly stronger intensities than those of the Ma and $\mathrm{Mb}$ peaks. The triclinic crystal structure is thus concluded to be the major component in 
the film after the HCFC-SVA treatment.

According to our former study, in a thin film, the monoclinic (Ma) phase has a thermodynamically stable structure; whereas the triclinic one has a metastable one [28], and no report is available for obtaining the triclinic one in a wet-processed film. The present study is thus the first report that the triclinic crystal is readily prepared by a 'wet process' in a thin film. This implies that ZnTPP has been made to have a low molecular mobility to be stabilized at the metastable structure by using HCFC as the solvent of SVA.

The diffraction pattern gives us another structural information of the 'crystal orientation' by referring the direction of the scattering vector, $q$, to that of the reciprocal lattice vector of the crystal structure. The $q_{x y}$ and $\sim q_{z}$ axis in Fig. 4 are parallel and normal to the substrate, respectively. As presented in Fig. $4 \mathrm{~b}$, the triclinic component has the 010 peak appeared mostly on the $q_{x y}$ axis $\left(q=0.50 \AA^{-1}\right)$; whereas the 100 peak appears roughly in the $\sim q_{z}$ axis direction with a slight shift of $\pm 6^{\circ}$ from the $\sim q_{z}$ axis $\left(q=0.63 \AA^{-1}\right)$. Since the reciprocal lattice vector has a direction vertical to the crystal plane, this result means that the $(010)$ plane is perpendicular to the substrate, and the (100) plane inclines $6^{\circ}$ from the substrate. The angle between the two planes is thus analyzed to be $84^{\circ}$ or $96^{\circ}$, which is consistent with the triclinic structure with the $c$ axis parallel to the surface as schematically drown in Fig. 5a. [27]. By rotating the schematic in Fig. 5a about the $z$ axis, 
the angle between the porphyrin ring and the substrate is found to be about $45^{\circ}$. Therefore, in the crystallized region, since the triclinic crystal is major component, the most of ZnTPP molecules should have this orientation.

Since a ZnTPP thin film can have an amorphous component [25] that does not appear in the GIXD pattern, the pMAIRS technique is employed for revealing the average molecular orientation of the entire region involving the crystal and the amorphous portions. In addition, the pMAIRS spectra are also correlated with the polymorph, and the key bands appear in the region of the $\gamma(\mathrm{C}-\mathrm{H})$ modes of a conjugate system [25].

Fig. 6 presents IR pMAIRS spectra of the as-spun and after HCFC-SVA films where the red and blue curves correspond to the IP and OP spectra, respectively. In the as-spun film, the amorphous structure is readily confirmed by four key bands at 798 , 752,719 and $700 \mathrm{~cm}^{-1}$ in Fig. $6 a$, which are known to be specific to the amorphous state (marked by 'A') [25,28]. In the HCFC-SVA film, on the other hand, the marker bands appear at different positions, and instead some characteristic bands of the triclinic phases (marked by ' $\mathrm{T}$ ') appear apparently accompanying monoclinic phases (marked by 'Ma') with a very minor intensity, as found in Fig. 6b. These characteristics basically agree with the crystal structures revealed by the 2D-GIXD analysis. 
Note that the $\gamma(\mathrm{C}-\mathrm{H})$ mode has a transition moment perpendicular to the porphyrin or phenyl ring [25]. The IP and OP bands of the as-spun film have comparable intensities for the four key bands (Fig. 6a), which indicates that the ZnTPP molecules are randomly oriented in the film [25]. After the HCFC-SVA treatment, the OP bands at 797 and $716 \mathrm{~cm}^{-1}$ are apparently larger than the IP ones (Fig. 6b) implying an oriented stance. Since these modes are localized on the porphyrin ring [25], the porphyrin ring is revealed to align parallel to the substrate, i.e., the face-on orientation on considering the surface selection rule of pMAIRS (see the Experimental section). The molecular orientation of ZnTPP in the thin films is analyzed quantitatively by using Eq. (1) as a function of the intensity ratio of the $\gamma(\mathrm{C}-\mathrm{H})$ bands in the pMAIRS-IP and -OP spectra. The orientation angle of the plane of the porphyrin ring from the substrate surface depicted in Fig. $5 \mathrm{~b}$ is calculated to be $43^{\circ}$ by using the intensity ratio of the $\gamma(\mathrm{C}-\mathrm{H})$ band at $797 \mathrm{~cm}^{-1}$. This angle agrees fairly well with the angle revealed by the 2D-GIXD analysis (ca. $45^{\circ}$ ). The quantitative agreement between the IR pMAIRS and the 2DGIXD techniques in terms of the molecular orientation apparently implies that the amorphous portion is ignorable in the film.

\section{Conclusion}


In the present study, the SVA treatment using HCFC-225 has readily made the ZnTPP film have a triclinic crystal structure without breaking the continuous surface topography, which cannot be attained by the thermal annealing or Chl-SVA, either. Another note is that the annealed film by HCFC-SVA has a metastable crystal structure as the major component. This result is understandable by considering the molecular mobility depending on the solvation by the solvent used in SVA. In the HCFC-SVA treatment, the ZnTPP molecules have a low mobility due to a weak solvation.

After the structural analysis in detail using 2D-GIXD and IR pMAIRS, the results of both polymorph and molecular orientation are found common to the two analytical techniques. In particular, the quantitative agreement of the molecular orientation of the porphyrin ring in the film has readily revealed that the film comprises the triclinic crystallite as the major component with a very minor component of the monoclinic crystallite, and ignorable portion of amorphous is found.

Acknowledgment: This work was financially supported by a Grant-in-Aid for Scientific Research (A) (No. 15H02185 (TH)), Grant-in-Aid for Young Scientists (B) (No. 17K14502 (TS)) and Grant-in-Aid for JSPSD fellows (No. 16J03487 (NS)) from the Japan Society for the Promotion of Science (JSPS), to which the authors' thanks are due. 
2D-GIXD measurements were carried out at BL46XU beam-line of SPring- 8 with the approval of the Japan Synchrotron Radiation Research Institute (JASRI) (Proposal No. 2017B1831). The authors appreciate Prof. Itaru Osaka for helping the GIXD measurements.

\section{References and Footnotes:}

1. Y. Wu, P. Liu, B.S. Ong, T. Srikumar, N. Zhao, G. Botton, S. Zhu, Controlled orientation of liquid-crystalline polythiophene semiconductors for high-performance organic thin-film transistors, Appl. Phys. Lett. $86 \quad$ (2005) 142102. doi:10.1063/1.1894597.

2. K. Abiko, Y. Kato, Analysis of the molecular orientation of poly(3-hexylthiophene) on silicon treated with silane coupling agents, by infrared p-polarized multiple-angle incidence resolution spectrometry, Chem. Lett. 47 (2018) 332-335. doi:10.1246/cl.171059.

3. Y. Diao, B.C.K. Tee, G. Giri, J. Xu, D.H. Kim, H.A. Becerril, R.M. Stoltenberg, T.H. Lee, G. Xue, S.C.B. Mannsfeld, Z. Bao, solution coating of large-area organic semiconductor thin films with aligned single-crystalline domains, Nat. Mater. 12 (2013) 665-671. doi:10.1038/nmat3650. 
4. K.C. Dickey, J.E. Anthony, Y.L. Loo, Improving organic thin-film transistor performance through solvent-vapor annealing of solution-processable triethylsilylethynyl anthradithiophene, Adv. Mater. 18 (2006) 1721-1726. doi:10.1002/adma.200600188.

5. W.Y. Chou, H.L. Cheng, An orientation-controlled pentacene film aligned by photoaligned polyimide for organic thin-film transistor applications, Adv. Funct. Mater. 14 (2004) 811-815. doi:10.1002/adfm.200305047.

6. C.R. Kagan, Organic-inorganic hybrid materials as semiconducting channels in thinfilm field-effect transistors, Science $286 \quad$ (1999) 945-947. doi:10.1126/science.286.5441.945.

7. R.R. Lunt, N.C. Giebink, A.A. Belak, J.B. Benziger, S.R. Forrest, Exciton diffusion lengths of organic semiconductor thin films measured by spectrally resolved photoluminescence quenching, J. Appl. Phys. $105 \quad$ (2009) 053711. doi:10.1063/1.3079797.

8. T. Komino, H. Tajima, M. Matsuda, A relationship between molecular orientation and current-voltage characteristics in poly(3-hexylthiophene) thin film, Chem. Lett. 37 (2008) 690-691. doi:10.1246/cl.2008.690. 
9. D. Shukla, S.F. Nelson, D.C. Freeman, M. Rajeswaran, W.G. Ahearn, D.M. Meyer, J.T. Carey, Thin-film morphology control in naphthalene-diimide-based semiconductors: high mobility n-type semiconductor for organic thin-film transistors, Chem. Mater. 20 (2008) 7486-7491. doi:10.1021/cm802071w.

10. A.L. Briseno, J. Aizenberg, Y.J. Han, R.A. Penkala, H. Moon, A.J. Lovinger, C. Kloc, Z. Bao, Patterned growth of large oriented organic semiconductor single crystals on self-assembled monolayer templates, J. Am. Chem. Soc. 127 (2005) 12164-12165. doi:10.1021/ja052919u.

11. K. Togashi, Y. Sagara, T. Yasuda, C. Adachi, Molecular design of high-molecularorientation electron-transport materials and application to organic light-emitting diodes, Chem. Lett. 42 (2013) 651-653. doi:10.1246/cl.130150.

12. F. Zhang, Y. Hu, T. Schuettfort, C. Di, X. Gao, C.R. Mcneill, L. Thomsen, S.C.B. Mannsfeld, W. Yuan, H. Sirringhaus, D. Zhu, Critical role of alkyl chain branching of organic semiconductors in enabling solution-processed n-channel organic thinfilm transistors with mobility of up to $3.50 \mathrm{~cm}^{2} \mathrm{~V}^{-1} \mathrm{~s}^{-1}$, J. Am. Chem. Soc. 135 (2013) 2338-2349. doi:10.1021/ja311469y.

13. B.R.J. Chesterfield, C.R. Newman, T.M. Pappenfus, P.C. Ewbank, M.H. Haukaas, K.R. Mann, L.L. Miller, C.D. Frisbie, High electron mobility and ambipolar transport 
pi-stacking quinoidal terthiophene, Adv. Mater. 15 (2003) 1278-1282. doi:10.1002/adma.200305200.

14. H.L. Cheng, J.W. Lin, Controlling polymorphic transformations of pentacene crystal through solvent treatments: an experimental and theoretical study, Cryst. Growth Des. 10 (2010) 4501-4508. doi:10.1021/cg100760t.

15. R.R. Lunt, J.B. Benziger, S.R. Forrest, Relationship between crystalline order and exciton diffusion length in molecular organic semiconductors, Adv. Mater. 22 (2010) 1233-1236. doi:10.1002/adma.200902827.

16. B.A. Gregg, Evolution of photophysical and photovoltaic properties of perylene bis(phenethylimide) films upon solvent vapor annealing, J. Phys. Chem. 100 (1996) 852-859. doi:10.1021/jp952557k.

17. E. Verploegen, C.E. Miller, K. Schmidt, Z. Bao, M.F. Toney, Manipulating the morphology of P3HT-PCBM bulk heterojunction blends with solvent vapor annealing, Chem. Mater. 24 (2012) 3923-3931. doi:10.1021/cm302312a.

18. C.D. Wessendorf, G.L. Schulz, A. Mishra, P. Kar, I. Ata, M. Weidelener, M. Urdanpilleta, J. Hanisch, E. Mena-Osteritz, M. Lindén, E. Ahlswede, P. Bäuerle, Efficiency improvement of solution-processed dithienopyrrole-based A-D-A 
oligothiophene bulk-heterojunction solar cells by solvent vapor annealing, Adv. Energy Mater. 4 (2014) 1-10. doi:10.1002/aenm.201400266.

19. H. Tang, G. Lu, L. Li, J. Li, Y. Wang, X. Yang, Precise construction of PCBM aggregates for polymer solar cells via multi-step controlled solvent vapor annealing, J. Mater. Chem. 20 (2010) 683-688. doi:10.1039/B917533D.

20. D.J. Mascaro, M.E. Thompson, H.I. Smith, V. Bulović, Forming oriented organic crystals from amorphous thin films on patterned substrates via solvent-vapor annealing, Org. Electron. Phys. Mater. Appl. 6 (2005) 211-220. doi:10.1016/j.orgel.2005.07.001.

21. D. Chirvase, J. Parisi, J.C. Hummelen, V. Dyakonov, Influence of nanomorphology on the photovoltaic action of polymer-fullerene composites, Nanotechnology 15 (2004) 1317-1323. doi:10.1088/0957-4484/15/9/035.

22. M. Manceau, A. Rivaton, J.L. Gardette, S. Guillerez, N. Lemaître, The mechanism of photo- and thermo-oxidation of poly(3-hexylthiophene) (P3HT) reconsidered, Polym. Degrad. Stab. $\quad 94 \quad$ (2009) 898-907. doi:10.1016/j.polymdegradstab.2009.03.005. 
23. F. C. Krebs, K. Norrman, Analysis of the failure mechanism for a stable organic photovoltaic during 10,000 h of testing, Prog. Photovolt: Res. Appl. 15 (2007) 697712. doi:10.1002/pip.794.

24. Y. Li, S.P. Singh, P. Sonar, A high mobility P-type DPP-thieno[3,2-b]thiophene copolymer for organic thin-film transistors, Adv. Mater. 22 (2010) 4862-4866. doi:10.1002/adma.201002313.E.

25. M. Hada, N. Shioya, T. Shimoaka, K. Eda, M. Hada, T. Hasegawa, Comprehensive understanding of structure-controlling factors of a zinc tetraphenylporphyrin thin film using pMAIRS and GIXD techniques, Chem. Eur. J. 22 (2016) 16539-16546. doi:10.1002/chem.201603291.

26. M.P. Byrn, C.J. Curtis, Y. Hsiou, S.I. Khan, P.A. Sawin, S.K. Tendick, C.E. Strouse, A. Terzis, Porphyrin sponges: conservation of host structure in over 200 porphyrinbased lattice clathrates, J. Am. Chem. Soc. 115 (1993) 9480-9497. doi:10.1021/ja00074a013.

27. W.R. Scheidt, J.U. Mondal, C.W. Eigenbrot, A. Adler, L.J. Radonovich, J.L. Hoard, Crystal and molecular structure of the silver(ii) and zinc(ii) derivatives of mesotetraphenylporphyrin. an exploration of crystal-packing effects on bond distance, Inorg. Chem. 25 (1986) 795-799. doi:10.1021/ic00226a014. 
28. N. Shioya, M. Hada, T. Shimoaka, R. Murdey, K. Eda, T. Hasegawa, Impact of kinetically restricted structure on thermal conversion of zinc tetraphenylporphyrin thin films to the triclinic and monoclinic phases, J. Phys. Chem. C. 122 (2018) 45404545. doi:10.1021/acs.jpcc.8b00972.

29. M. Yoneya, A. Miyamoto, Y. Shimizu, M. Ohmori, A. Fujii, M. Ozaki, Characterization of crystal polymorphs of the organic semiconductor non-peripheral octahexyl phthalocyanine characterization of crystal polymorphs of the organic semiconductor, Jpn. J. Appl. Phys. $56 \quad$ (2017) 081601 doi.org/10.7567/JJAP.56.081601.

30. J. Kim, S, Yim, Phase Transition of $\mathrm{ZnPc}$ thin films via thermal annealing, and its effect on the performance of $\mathrm{ZnPc} / \mathrm{C}-60$ photovoltaic cells J. Nanoelectron. Optoelectron. 6 (2011) 249. doi.org/10.1166/jno.2011.1162.

31. T. Hasegawa, Physicochemical nature of perfluoroalkyl compounds induced by fluorine, Chem. Rec. 17 (2017) 903-917. doi:10.1002/tcr.201700018.

32. S.T. Wellinghoff, J.L. Koenig, E. Baer, Spectroscopic examination of chain conformation and bonding in poly (phenylene oxide)-polystyrene, J. Polym. Sci. Polym. Phys. Ed. 15 (1977) 1913-1925. doi:10.1002/pol.1977.180151104. 
33. F.M. Fowkes, Role of acid-base interfacial bonding in adhesion, J. Adhes. Sci. Technol. 1 (1987) 7-27. doi:10.1163/156856187X00049.

34. K.J. Miller, Additivity methods in molecular polarizability, J. Am. Chem. Soc. 112 (1990) 8533-8542. doi:10.1021/ja00179a044.

35. T. Hasegawa, Advanced multiple-angle incidence resolution spectrometry for thinlayer analysis on a low-refractive-index substrate, Anal. Chem. 79 (2007) 4385-4389. doi: $10.1021 / \mathrm{ac} 070676 \mathrm{~d}$.

36. T. Hasegawa, A new approach to analysis of molecular structure in thin films: infrared multiple-angle incidence resolution spectrometry, Appl. Spectrosc. Rev. 43 (2008) 181-201. doi: 10.1080/05704920801944312.

37. T. Hasegawa, Quantitative infrared spectroscopy for understanding of a condensed matter, Springer, Tokyo, 2017. doi: 10.1007/978-4-431-56493-5.

38. N. Shioya, S. Norimoto, N. Izumi, M. Hada, T. Shimoaka, T. Hasegawa, Optimal experimental condition of IR pMAIRS calibrated by using an optically isotropic thin film exhibiting the Berreman Effect, Appl. Spectrosc. 71 (2017) 901-910. doi:10.1177/0003702816658673. 
39. T. Hasegawa, A novel measurement technique of pure out-of-plane vibrational modes in thin films on a nonmetallic material with no polarizer, J. Phys. Chem. B. 106 (2002) 4112-4115. doi:10.1021/jp013751b.

40. T. Hasegawa, A new spectroscopic tool for surface layer analysis: multiple-angle incidence resolution spectrometry, Anal. Bioanal. Chem. 388 (2007) 7-15. doi:10.1007/s00216-006-1116-8.

41. E.M. Han, L.M. Do, M. Fujihira, H. Inada, Y. Shirota, Scanning force microscopy of organic thin-film amorphous hole transport materials, J. Appl. Phys. 80 (1996) 32973305. doi:10.1063/1.363239.

42. A.J. Moulé, K. Meerholz, Morphology control in solution-processed bulkheterojunction solar cell mixtures, Adv. Funct. Mater. 19 (2009) 3028-3036. doi:10.1002/adfm.200900775.

M.P. Byrn, C.J. Curtis, Y. Hsiou, S.I. Khan, P.A. Sawin, S.K. Tendick, C.E. Strouse, A. Terzis, Porphyrin sponges: conservation of host structure in over 200 porphyrinbased lattice clathrates, J. Am. Chem. Soc. 115 (1993) 9480-9497. doi:10.1021/ja00074a013. 


\section{Figure captions:}

Fig. 1 AFM images of (a) as-spun film, (b) after thermal annealing at $100{ }^{\circ} \mathrm{C}$, and (c) SVA using Chl.

Fig. 2 (a) Triclinic and (b) monoclinic crystal structures of ZnTPP.

Fig. 3 AFM images of (a) a silicon substrate before spin-coating, (b) as-spun (rms = $0.38 \mathrm{~nm})$, and (c) after SVA using HCFC-225 (rms $=26 \mathrm{~nm})$.

Fig. 4 2D-GIXD patterns of ZnTPP thin films for (a) as-spun, and (b) SVA treated using HCFC-225. ' $\mathrm{T}$ ' and 'Ma', 'Mb' represent the peak of a triclinic and two different monoclinic phases, respectively.

Fig. 5 The schematics of molecular orientation of the triclinic crystallite from the parallel viewpoint to the substrate plane. (a) The $\mathrm{c}$ axis is aligned parallel to the viewpoint direction. (b) On the same crystallite, the viewpoint direction is rotated about the $\mathrm{z}$ axis, so that the porphyrin planes are made parallel to the direction.

Fig. 6 IR pMAIRS spectra in the $\gamma(\mathrm{C}-\mathrm{H})$ region of (a) the as-spun film, and (b) the film after HCFC-SVA. The red and blue curves correspond to the IP and OP spectra respectively. 
(a)

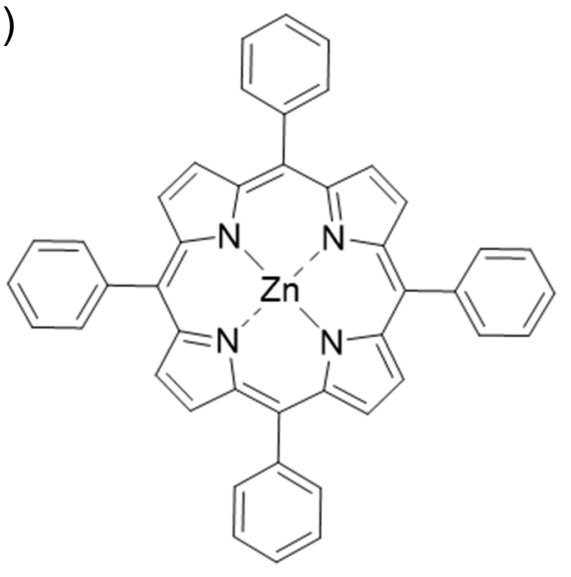

(c)

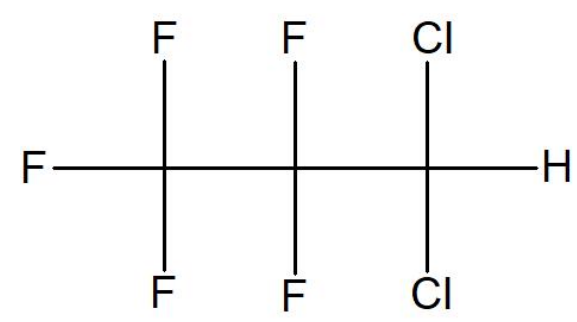

(b)<smiles>ClC(Cl)Cl</smiles>

Chart 1 Chemical structures of (a) zinc tetraphenylporphyrin (ZnTPP), (b) Chloroform (Chl) and (c) HCFC-225, which is the mixture of HCFC-225ca (left) and HCFC-225cb (right). 
(a) as-spun

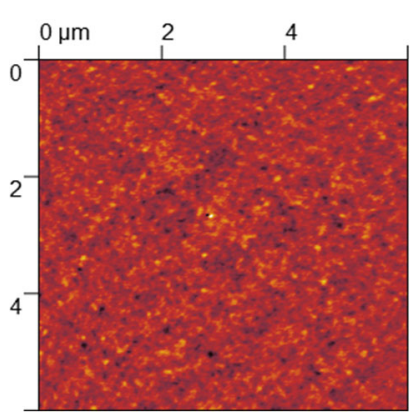

(b) after thermal annealing

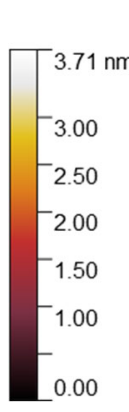

(c) after Chl-SVA

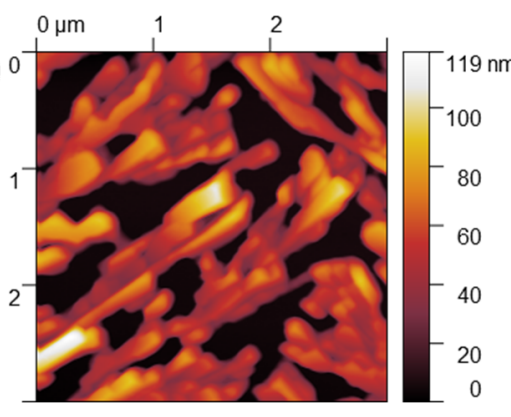

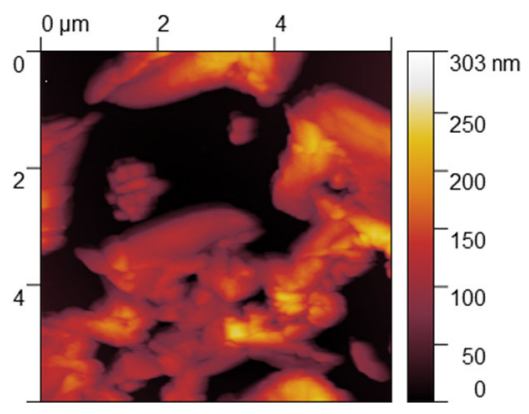

Fig. $1 \mathrm{~K}$. Tomita et al.

(a)

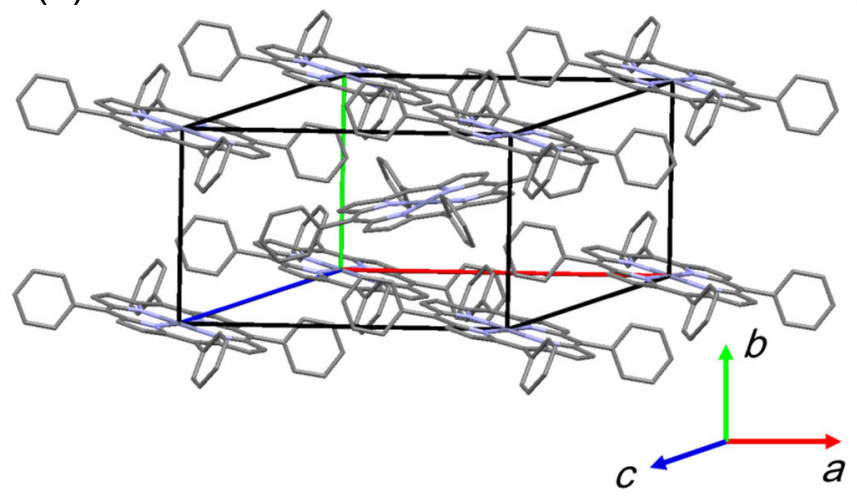

(b)

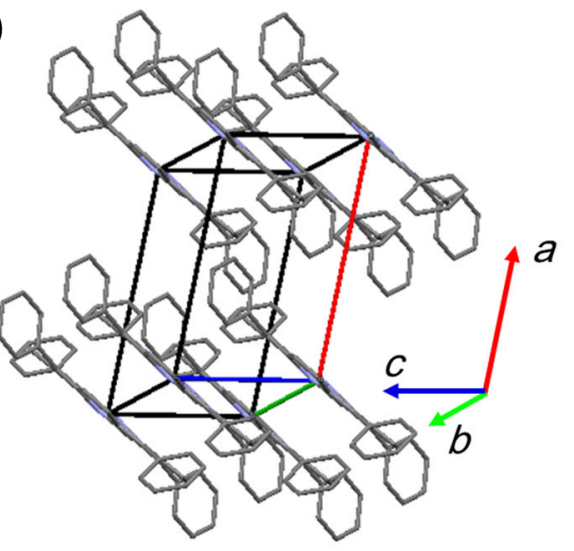

Fig. 2 K. Tomita et al. 
(a) silicon

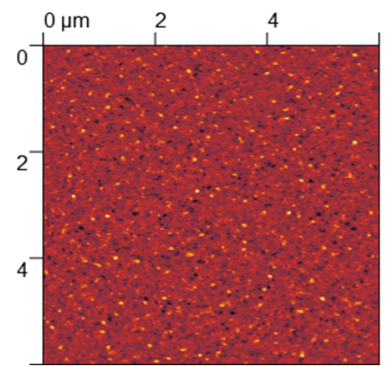

(b) as-spun

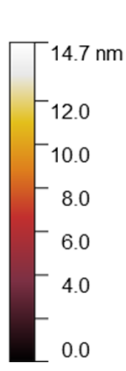

(c) after HCFC-SVA

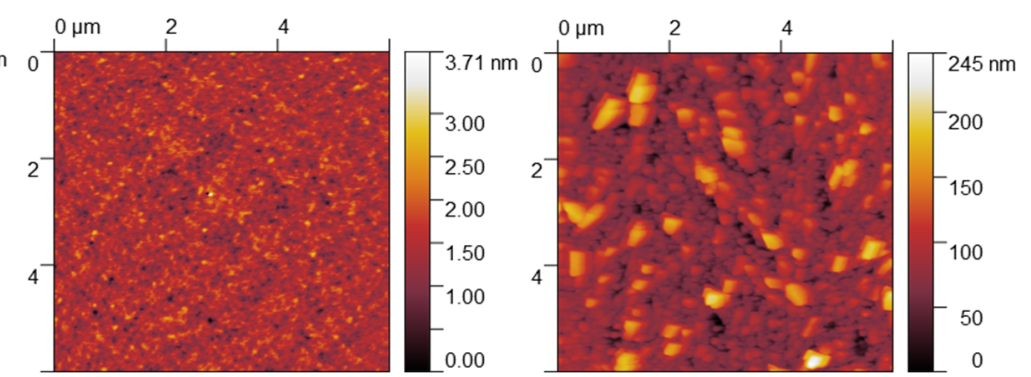

Fig. 3 K. Tomita et al. 
(a) as-spun

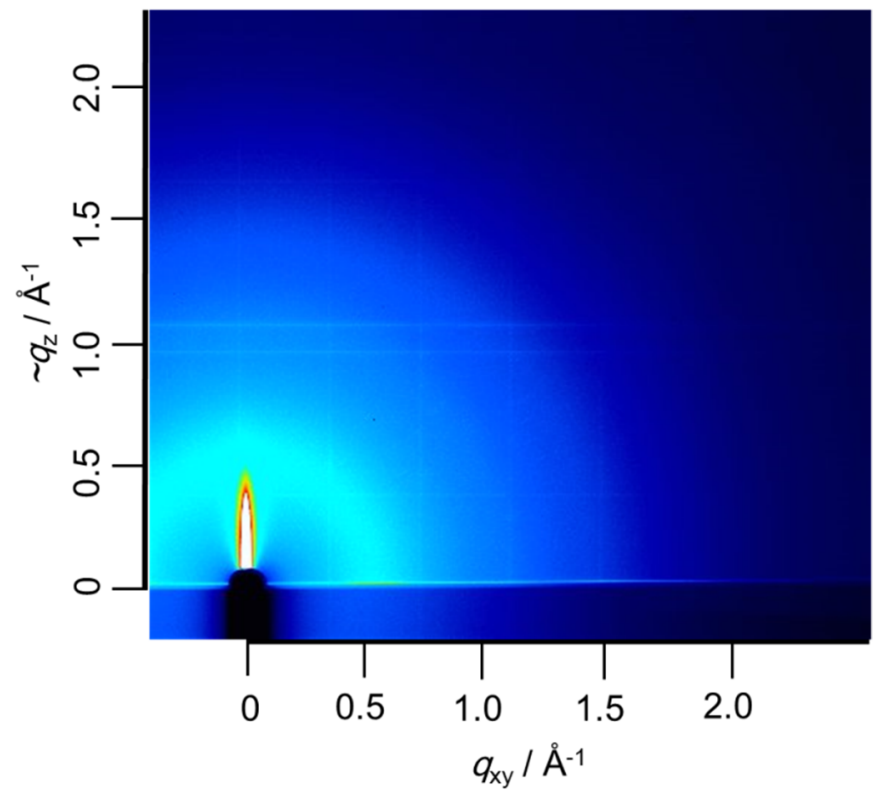

(a) after HCFC-SVA

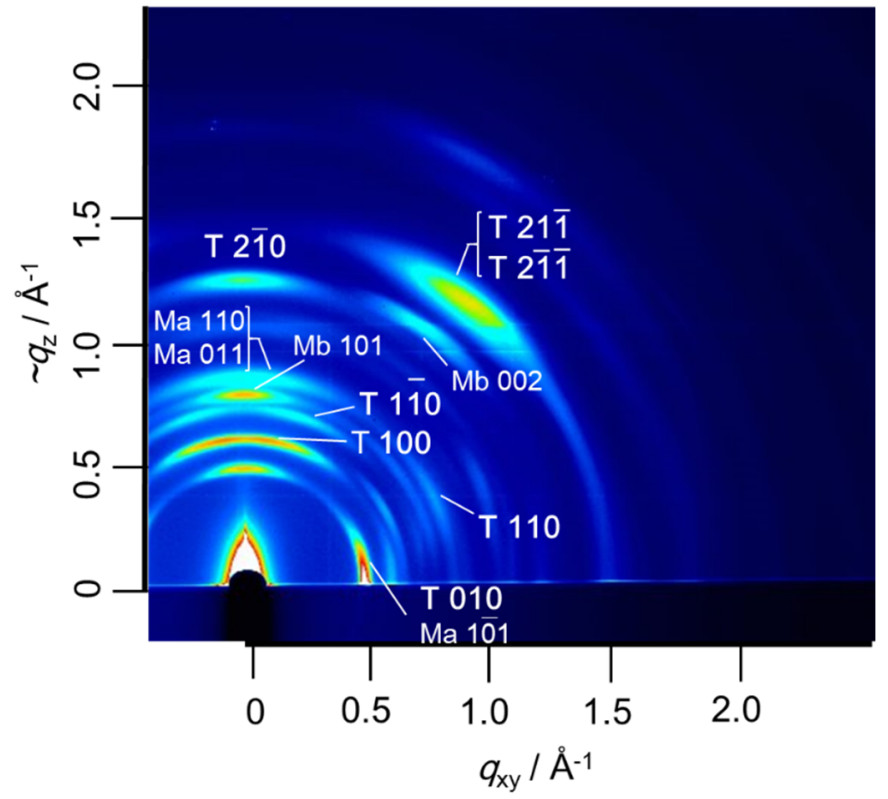

Fig. 4 K. Tomita et al. 
(a)

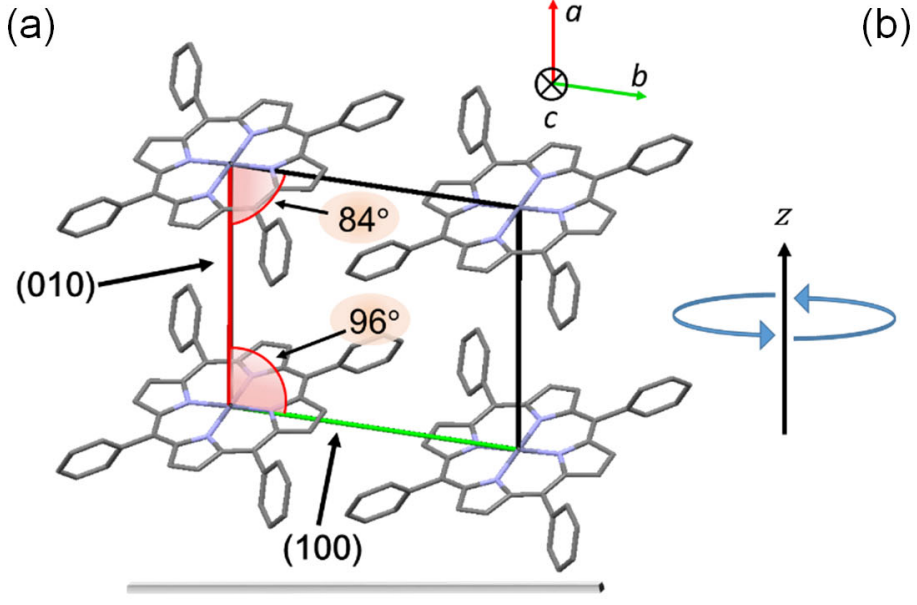

(b)

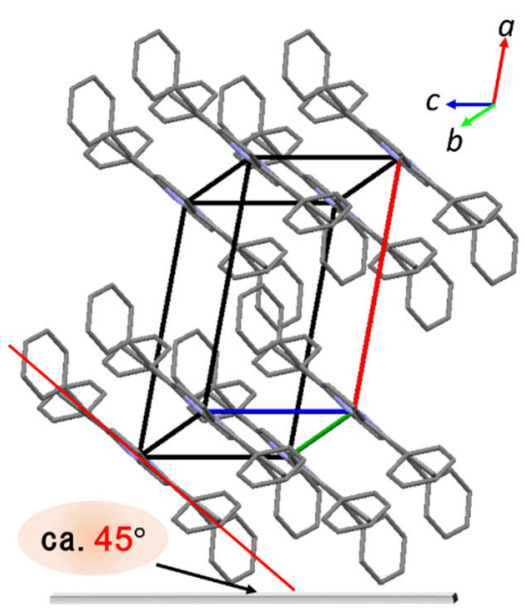

Fig. 5 K. Tomita et al. 


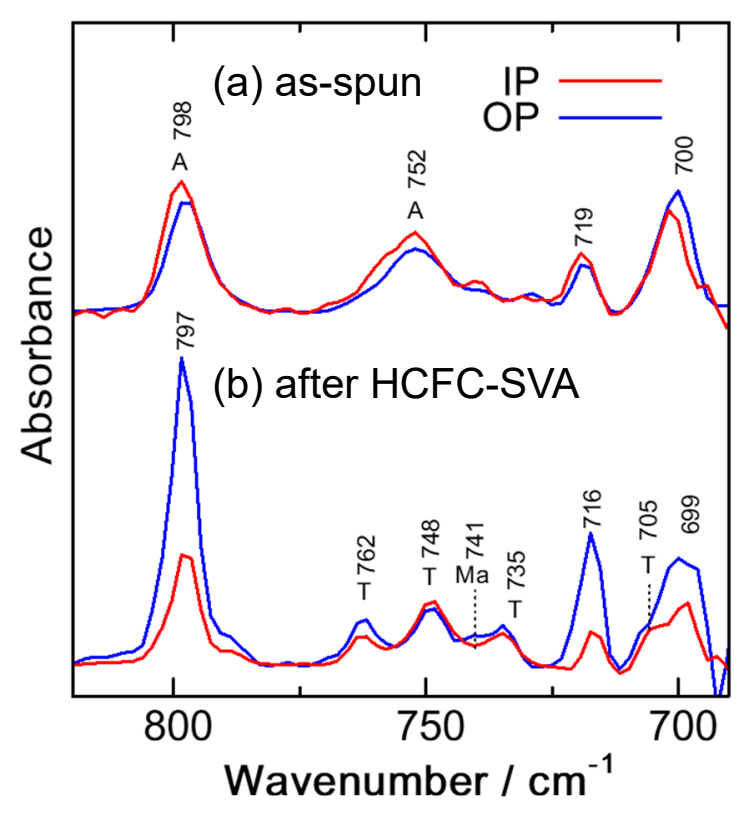

Fig. 6 K. Tomita et al. 